\title{
Neutrophil-lymphocyte ratio (NLR) and platelet-lymphocyte ratio (PLR) may be superior to C-reactive protein (CRP) for predicting the occurrence of differentiated thyroid cancer
}

\author{
${ }^{1}$ Ozmen S, ${ }^{2}$ Timur O, ${ }^{1}$ Calik I, ${ }^{3}$ Altinkaynak K, ${ }^{4}$ Simsek E, ${ }^{2}$ Gozcu H, ${ }^{2}$ Arslan A, ${ }^{5}$ Carlioglu A
}

\author{
${ }^{1}$ Department of Pathology, Erzurum Training and Research Hospital, Erzurum, Turkey \\ ${ }^{2}$ Department of Internal Medicine, Erzurum Training and Research Hospital, Erzurum, Turkey \\ ${ }^{3}$ Department of Biochemistry, Erzurum Training and Research Hospital, Erzurum, Turkey \\ ${ }^{4}$ Department of Otorhinolaryngology, Erzurum Training and Research Hospital, Erzurum, Turkey \\ ${ }^{5}$ Department of Endocrinology, Erzurum Training and Research Hospital, Erzurum, Turk \\ E-mail: ozgetimur@yahoo.com
}

Objectives. NLR (neutrophil-lymphocyte ratio) and PLR (platelet-lymphocyte ratio) are prognostic markers of differentiated thyroid cancers. In our study, we evaluated NLR, PLR and C-reactive protein (CRP) for predicting the occurence of differentiated thyroid cancer. This is the first study that compares NLR and PLR to C-reactive protein indifferantiated thyroid cancer not only papillary cancer but also folliculer cancer.

Methods. This study includes 51 papillary carcinoma, 42 papillary microcarcinoma and 31 folliculer carcinoma patients attending to our outpatient Endocrinology Clinic at Erzurum Region Training and Research Hospital between 2009 and 2014. The control group include 50 age, sex and body mass index matched healty subjects. Blood counts and CRP were measured at the day before surgery. Thyroglobulin was measured after 6 months of operation.

Results. There were positive correlations between tumor diameter, age, white blood cell (WBC) and thyroglobulin levels. There were also positive correlation between NLR, PLR and CRP levels.

Conclusion. In our study, we found out that higher NLR and PLR was associated with higher levels of thyroglobulin which indicates worse survival. CRP levels were also associated with poorer tumor profile but the determining rate was lower according to ROC analysis

Key words: neutrophil-lymphocyte ratio, platelet-lymphocyte ratio, C-reactive protein, differentiated thyroid cancer

Papillary and follicular cancers are considered to be differentiated cancers and patients with these tumors are often treated similarly despite the numerous biologic differences. Most anaplastic (undifferentiated) cancers appear to arise from the differentiated ones (Tuttle 2017). Papillary thyroid carcinoma is the most common type of the differentiated thyroid carcinoma (Lang et al. 2014). Despite its relatively good prognosis with a 10 -year, cancer specific survival $>90 \%$, local, and regional recurrence is common (Lang et al. 2007).

There is an increasing and consistent evidence that the inflammation is related to the cancer development and progression (Coussens and Werb 2002; Whiteside 2008; Nozawa et al. 2006). Systemic inflammatory markers, including $\mathrm{C}$-reactive protein, albumin

Corresponding author: Dr. Ozge Timur, Erzurum Region Training and Research Hospital, Department of Internal Medicine, Ataturk Mahallesi Cat Yolu Caddesi 25040 Yakutiye, Erzurum, Turkey; phone: 0505 8574627; e-mail: ozgetimur@yahoo.com. 
concentration, and neutrophil to lymphocyte ratio (NLR) have recently been shown to be independent prognostic factors in the cancer patients (McMillan 2013; Guthrie et al. 2013). The NLR has been shown to be a convenient and inexpensive prognostic biomarker in many human cancers (Azab et al. 2012; Kishi et al. 2009; Wang et al. 2011). Platelet to lymphocyte ratio (PLR) is a novel marker. Platelet counts as an inflammation marker and is involved in the progress of tumor invasion (Kim et al. 2014). These markers can be easily, inexpensively, and reproducibly assayed, enhancing the ability to evaluate the prognosis in the cancer patients.

Many studies have reported that inflammation is associated with the cancer prognosis. Unlike other types, few studies have reported about the thyroid cancer. Seretis et al. (2013) have compared the NLR between with an incidental papillary microcarcinoma and those with a benign goiter only and found that NLR was significantly higher in carcinoma patients. Liu et al. (2013) have studied the correlation between the preoperative NLR and the tumor characteristics. They have found that NLR is significantly higher with large sized tumors.

Kim et al. (2014) have reported the associations of NLR with disease-free and overall survival. They have found out that higher NLR was a negative prognostic marker for disease-free survival.

In the view of these findings, NLR and PLR are prognostic markers of differentiated thyroid cancers. In our study, we evaluated NLR, PLR, and C-reactive protein for predicting the occurrence of differentiated thyroid cancer. This is the first study that compares NLR and PLR to C-reactive protein in differentiated thyroid cancer not only the papillary cancer but also the follicular one.

\section{Subjects and Methods}

Subjects. This study includes 51 papillary carcinoma, 42 papillary microcarcinoma, and 31 follicular carcinoma patients attending our outpatient Endocrinology Clinic at Erzurum Region Training and Research Hospital between 2009 and 2014. The control group includes 50 -age, sex and body mass index matched healthy subjects. The control group was chosen between post operative benign nodular goiter patients. All the differential thyroid carcinoma patients were diagnosed histopathologically. Blood counts and CRP were measured at the day before surgery. Thyroglobulin was measured 6 months after an operation. Ethics Committee of Erzurum Region Training and Research Hospital approved the study design. All the study subjects provided written informed consent.

Laboratory assessment. Individual types of the differentiated cancers were evaluated histopathologically from the post-operative samples by pathology department of Erzurum Region Training and Research Hospital.

Neutrophil to lymphocyte ratio (NLR) and platelet to lymphocyte ration (PLR) were calculated from full blood count. The Beckman Coulter LH750 (impedance method) analyzer was used for complete blood counts.

Thyroglobulin and C-reactive protein (CRP) levels were measured by standard laboratory methods on a biochemistry autoanalyzer (Beckman Coulter AU 2700 Plus clinical chemistry autoanalyzer) with the company's original kits (CRP N: 0-1 mg/dl).

Statistical analysis. Data are presented as mean \pm SD. The IBM SPSS Statistics version 17 was used for statistical analysis. Student's t-test was done to find the significance of difference between means whenever applicable. One tailed Pearson's correlation test was done to find the correlation between various variables. Linear regression analysis was done whenever appropriate. Chi square test, Chi square with Yates correction and Fisher's exact test, wherever applicable, were used to test the association between two findings. The receiver operating characteristic (ROC) curve analysis assessed the cut-off of NLR and PLR with the best diagnostic accuracy for detecting differentiated thyroid cancers.

\section{Results}

NLR, PLR and CRP levels and demographic features of the all groups are shown in Table 1 . As each group was compared with other control group levels were significantly lower than other cancer groups ( $\mathrm{p}>0.05)$.

Thyroglobulin levels were measured at the $6^{\text {th }}$ month. The results are as following: $11.32 \pm 51.50 \mathrm{ng} / \mathrm{ml}$ in papillary cancer, $0.84 \pm 2.93 \mathrm{ng} / \mathrm{ml}$ in papillary $\mathrm{mi}$ crocancer, $58.78 \pm 106.68 \mathrm{ng} / \mathrm{ml}$ in follicular cancer, and $1.15 \pm 2.65 \mathrm{ng} / \mathrm{ml}$ in control group. As thyroglobulin levels of each group compared with other follicular cancer group result was significantly higher than control, papillary and papillary microcancer group $(\mathrm{p}=0.002, \mathrm{p}=0.009$ and $\mathrm{p}=0.000$; respectively).

To assess the correlation between NLR and PLR, a Pearson correlation analysis was performed on each variable (Table 2). There were positive correlations between tumor diameter, age, white blood cell (WBC) and thyroglobulin levels. There were also positive correlations between NLR, PLR and CRP levels. 
Table 1

NLR, PLR, CRP levels and demographic features of each groups.

\begin{tabular}{|c|c|c|c|c|}
\hline Parameters & Papillary Cancer & Papillary Microcancer & Follicular Cancer & Control \\
\hline $\mathrm{N}$ & 51 & 42 & 31 & 50 \\
\hline Age & $37.7 \pm 15.2$ & $43.9 \pm 10.2$ & $44.5 \pm 11.6$ & $46.5 \pm 13.0$ \\
\hline BMI & $25.44 \pm 6.68$ & $28.32 \pm 4.32$ & $27.84 \pm 5.30$ & $27.18 \pm 4.83$ \\
\hline Neutrophil $\left(10^{3} / \mu \mathrm{l}\right)$ & $4.00 \pm 1.31$ & $5.81 \pm 2.42$ & $5.93 \pm 2.83$ & $5.53 \pm 3.22$ \\
\hline Lymphocyte $\left(10^{3} / \mu \mathrm{l}\right)$ & $2.60 \pm 0.70$ & $2.01 \pm 0.77$ & $1.94 \pm 0.67$ & $1.87 \pm 0.61$ \\
\hline Platelets $\left(10^{3} / \mu \mathrm{l}\right)$ & $258.66 \pm 60.62$ & $266.74 \pm 68.20$ & $251.31 \pm 57.96$ & $266.61 \pm 66.03$ \\
\hline $\mathrm{WBC}(103 / \mu \mathrm{l})$ & $7.22 \pm 1.52$ & $8.65 \pm 2.86$ & $8.64 \pm 2.83$ & $8.12 \pm 3.17$ \\
\hline NLR & $3.10 \pm 1.85$ & $2.85 \pm 1.39$ & $3.55 \pm 2.91$ & $1.62 \pm 0.56$ \\
\hline PLR & $154.41 \pm 76.49$ & $141.23 \pm 63.44$ & $163.13 \pm 88.14$ & $103.12 \pm 33.75$ \\
\hline CRP (mg/dl) & $13.24 \pm 39.97$ & $3.79 \pm 4.48$ & $5.37 \pm 7.31$ & $3.09 \pm 5.71$ \\
\hline Waist Circumference $(\mathrm{cm})$ & $97.00 \pm 7.65$ & $93.86 \pm 12.03$ & - & $81.40 \pm 11.03$ \\
\hline Fasting Glucose(mg/dl) & $97.50 \pm 24.79$ & $97.31 \pm 15.77$ & $103.37 \pm 64.79$ & $87.76 \pm 7.86$ \\
\hline Urea $(\mathrm{mg} / \mathrm{dl})$ & $31.81 \pm 11.89$ & $29.10 \pm 7.57$ & $29.51 \pm 8.77$ & $31.81 \pm 8.81$ \\
\hline Creatine (mg/dl) & $1.09 \pm 2.35$ & $0.70 \pm 0.15$ & $0.69 \pm 0.16$ & $0.80 \pm 0.17$ \\
\hline $\operatorname{AST}(\mathrm{mg} / \mathrm{dl})$ & $23.72 \pm 6.72$ & $20.02 \pm 6.16$ & $21.48 \pm 5.61$ & $20.10 \pm 5.61$ \\
\hline $\operatorname{ALT}(\mathrm{mg} / \mathrm{dl})$ & $23.00 \pm 12.08$ & $19.5 \pm 9.37$ & $20.09 \pm 9.88$ & $18.77 \pm 9.89$ \\
\hline GGT (mg/dl) & $22.02 \pm 15.52$ & $14.71 \pm 5.56$ & $21.03 \pm 12.94$ & $17.27 \pm 9.39$ \\
\hline $\operatorname{ALP}(\mathrm{mg} / \mathrm{dl})$ & $185.00 \pm 77.36$ & $176.64 \pm 69.10$ & $203.14 \pm 68.30$ & $242.47 \pm 227.61$ \\
\hline Total Cholesterol (mg/dl) & $199.26 \pm 49.66$ & $201.13 \pm 38.79$ & $192.73 \pm 44.38$ & $176.58 \pm 45.08$ \\
\hline Triglycerides (mg/dl) & $162.37 \pm 79.23$ & $162.02 \pm 60.52$ & $130.00 \pm 64.07$ & $101.77 \pm 46.74$ \\
\hline Uric Acid (mg/dl) & $4.11 \pm 0.98$ & $4.00 \pm 1.27$ & $4.27 \pm 1.36$ & $4.22 \pm 1.26$ \\
\hline
\end{tabular}

Abbreviations: NLR - neutrophil-lymphocyte ratio; PLR - platelet-lymphocyte ratio; CRP - C-reactive protein; BMI - body mass index; WBC - white blood cells

Table 2

Correlation coefficients determined by simple correlation between the NLR and other clinical factors.

\begin{tabular}{lcc}
\hline Variables & $\begin{array}{c}\text { Correlation } \\
\text { Coefficient }\end{array}$ & p-value \\
\hline Tumor Diameter & 0.219 & 0.03 \\
PLR & 0.610 & 0.00 \\
Age & 0.234 & 0.002 \\
WBC & 0.571 & 0.00 \\
Tg & 0.261 & 0.02 \\
CRP & 0.290 & 0.01 \\
\hline
\end{tabular}

Abbreviations: NLR - neutrophil-lymphocyte ratio; PLR - platelet-lymphocyte ratio; WBC - white blood cells; Tg thyroglobulin; CRP - C-reactive protein

The multiple regression analysis of NLR and other risk factors was performed (Table 3). Age, gender, $\mathrm{BMI}$ and CRP were independent predictive factors of NLR. Adjustment for other these factors did not alter these relative risks.
Table 3

Multiple regression analysis of clinical factors possibly affecting the NLR in thyroid differentiated cancer subjects' adjusted forage.

\begin{tabular}{lcc}
\hline Variables & $\boldsymbol{\beta}$ & p-value \\
\hline NLR & 0.117 & $0.008^{*}$ \\
Age & 0.010 & 0.083 \\
Gender & -0.28 & 0.085 \\
BMI & -0.03 & 0.833 \\
CRP & -0.09 & 0.578 \\
\hline
\end{tabular}

Abbreviations: NLR - neutrophil-lymphocyte ratio; BMI body mass index; CRP - C-reactive protein

NLR and PLR were more successful in diagnosing thyroid cancer than CRP, which has ACU value 0.58 $(\mathrm{p}=0.28)$. AUC value of NLR was $0.831(\mathrm{p}=0.000)$ and PLR was $0.796(\mathrm{p}=0.000)$. The results of ROC analysis are shown in Figure 1. 


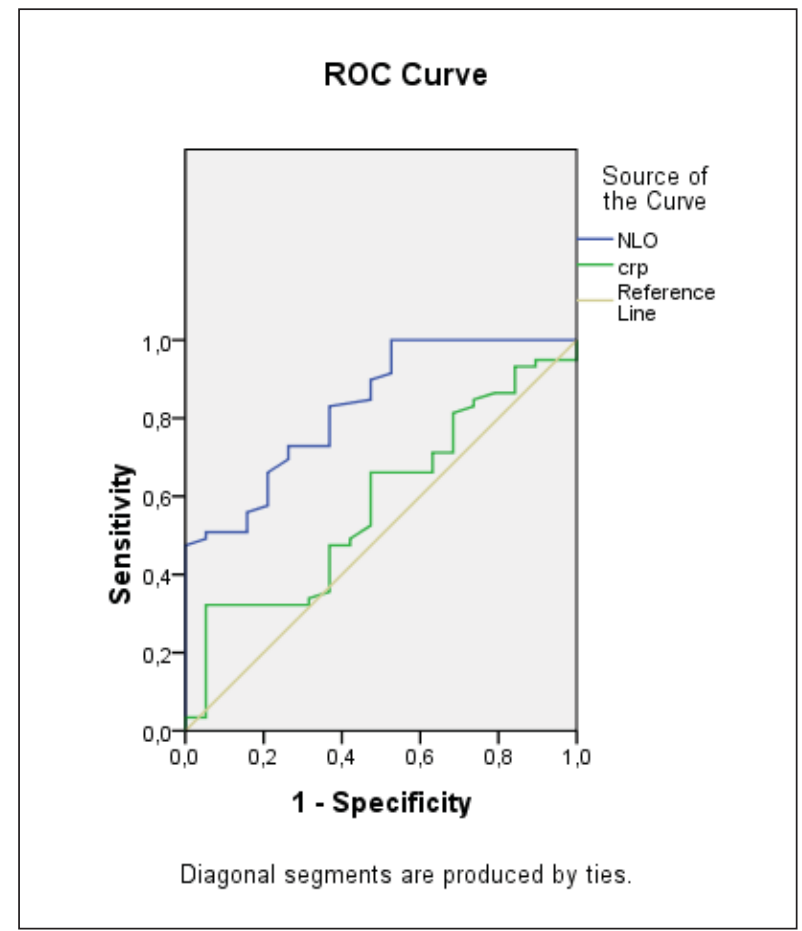

Figure 1. The ROC curve. AUC NLR: 0.831, AUC CRP: 0.581 and true area 0.5 .

\section{Discussion}

NLR is an independent prognostic factor of survival in patients with upper gastrointestinal cancers and several types of solid organ malignancies (Walsh et al. 2005; Jung et al. 2011; Chen et al. 2015; Duzlu et al. 2015; Langsenlehner et al. 2015; Okamura et al. 2015; Song et al. 2015; Szkandera et al. 2015; Ji et al. 2016). However, there are a few studies regarding the thyroid cancers.

Seretis et al. (2013) have observed that NLR was significantly elevated in patients with incidental papillary microcarcinoma and thyroid cancers.

In the study of Kim et al. (2014), prognostic importance of NLR with advanced papillary thyroid cancer have been described. They used different cutoff for NLR and found that despite heterogeneous cutoffs higher NLR was associated with more advanced stage of the disease and more aggressive tumor behavior.

Lang et al. (2007) have evaluated the preoperative NLR in papillary thyroid cancer. They have found that although higher NLR may imply a poorer tumor profile, it was not significantly associated with a worse disease free survival and higher risk of occult central nodal metastasis in papillary thyroid cancer.

In our study, both control and study group of patients had thyroid nodules. Thyroid nodule is one of the common diseases originating from the endocrine system. Most such nodules are benign, but 5-10\% have been reported to be malign. Differentiating the nodules is clinically important. A recent pilot study by Seretis et al. (2013) have shown that mean preoperative NLR was significantly higher in patients with than without papillary thyroid microcarcinoma and thyroid cancer. In contrast, in the study of Liu et al. (2013), NLR did not differ in patients with thyroid cancer and those with follicular adenoma. In the study of Kim et al. (2014), NLR was not an independent marker of thyroid malignancy but NLR was a marker for thyroid nodules, whether benign or malignant. They also have suggested that NLR tended to be higher in patients with solid than cystic thyroid nodules (Kim et al. 2014).

These few studies regarding the thyroid cancer have shown that higher NLR is associated with the poor prognosis. However, in our study, we evaluated NLR, PLR, and CRP. This is the first study to evaluate and compare all these parameters. In our study, we also compared different types of thyroid cancers. All the previous studies published were about the papillary cancers, however, our study also includes follicular cancers.

In our study, we found out that higher NLR and PLR was associated with higher levels of thyroglobulin, which indicates worse survival. CRP levels were also associated with poorer tumor profile but the determining rate was lower according to ROC analysis.

There are few studies comparing NLR and CRP predictive values. Forget et al. (2015) have found that NLR was significantly associated with postoperative complications of major abdominal surgery than CRP.

In the study of Turkmen et al. (2013), has been indicated that PLR may better predict the inflammation than NLR in the end stage of the renal disease. However, in our study, both NLR and PLR were better than CRP but NLR was also better than PLR. This difference could be because PLR count is an inflammation marker and is involved in the progress of the tumor invasion.

As NLR and PLR are simple biomarkers, they can be easily determined as well as easily reproducible. These facts make them great tools in the prognosis assessing probably more efficient than CRP and they can be used in various types of population to determine the thyroid cancer survival and risk of recurrence.

In summary, we found that NLR and PLR are superior to CRP in the predicting of the occurrence of differential thyroid cancer. Further studies are needed to evaluate long term survival, risk of recurrence, and disease free stage. 


\section{References}

Azab B, Bhatt VR, Phookan J, Murukutla S, Kohn N, Terjanian T, Widmann WD. Usefulness of the neutrophil-tolymphocyte ratio in predicting short- and long-term mortality in breast cancer patients. Ann Surg Oncol 19, 217-224, 2012.

Chen Q, Yang LX, Li XD, Yin D, Shi SM, Chen EB, Yu L, Zhou ZJ, Zhou SL, Shi YH, Fan J, Zhou J, Dai Z. The elevated preoperative neutrophil-to-lmyphocyte ration predicts poor prognosis in intrahepatic cholangiocarcinoma patients undergoing hepatectomy. Tumour Biol 36, 5283-5289, 2015.

Coussens LM, Werb Z. Inflammation and cancer. Nature 420, 860-867, 2002.

Duzlu M, Karamert R, Tutar H, Karaloglu F, Sahin M, Cevizci R. Neutrophil-lymphocyte ratio findings and larynx carcinoma: a preliminary study in Turkey. Asian Pac J Cancer Prev 16, 351-354, 2015.

Forget P, Dinant V, De Kock M. Is the neutrophil-to-lymphocyte ratio more correlated than C-reactive protein with postoperative complications after major abdominal surgery? Peer J 3, e713, 2015.

Guthrie GJ, Charles KA, Roxburgh CS, Horgan PG, McMillan DC, Clarke SJ. The systemic inflammation-based neutrophil-lymphocyte ratio: experience in patients with cancer. Crit Rev Oncol Hematol 88, 218-230, 2013.

Ji WH, Jiang YH, Ji YL, Mao WM. Prechemotherapy neutrophil: lymphocyte ratio is superior to the platelet: lymphocyte ratio as a prognostic indicator for locally advanced esophageal squamous cell cancer treated with neoadjuvant chemotherapy. Dis Esophagus 29, 403-411, 2016.

Jung MR, Park YK, Jeong O, Seon JW, Ryu SY, Kim DY, Kim YJ. Elevated preoperative neutrophil to lymphocyte ratio predicts poor survival following resection in late stage gastric cancer. J Surg Oncol 104, 504-510, 2011.

Kim JY, Park T, Jeong SH, Jeong CY, Ju YT, Lee YJ, Hong SC, Ha WS, Choi SK, Jung EJ. Prognostic importance of baseline neutrophil to lymphocyte ratio in patients with advanced papillary thyroid carcinomas. Endocrine 46, 526-531, 2014.

Kishi Y, Kopetz S, Chun YS, Palavecino M, Abdalla EK, Vauthey JN. Blood neutrophil-to-lymphocyte ratio predicts survival in patients with colorectal liver metastases treated with systemic chemotherapy. Ann Surg Oncol 16, 614-622, 2009.

Lang B, Lo CY, Chan WF, Lam KY, Wan KY. Restaging of differentiated thyroid carcinoma by the sixth edition AJCC/UICC TNM staging system: stage migration and predictability. Ann Surg Oncol 14, 1551-1559, 2007.

Lang BH, Ng CP, Au KB, Wong KP, Wong KK, Wan KY. Does preoperative neutrophil lymphocyte ratio predict risk of recurrence and occult central nodal metastasis in papillary thyroid carcinoma? World J Surg 38, 2605-2612, 2014.

Langsenlehner T, Thurner EM, Krenn-Piko S, Langsenlehner U, Stojakovic T, Gerger A, Pichler M. Validation of the neutrophil-to-lymphocyte ration as a prognostic factor in an cohort of European prostate cancer patients. World J Urol 33, 1661-1667, 2015.

Liu CL, Lee JJ, Liu TP, Chang YC, Hsu YC, Cheng SP. Blood neutrophil-to-lymphocyte ration correlates with tumor size in patients with differentiated thyroid cancer. J Surg Oncol 107, 493-497, 2013.

McMillan DC. The systemic inflammation-based Glasgow prognostic score: a decade of experience in patients with cancer. Cancer Treat Rev 39, 534-540, 2013.

Nozawa H, Chiu C, Hanahan D. Infiltrating neutrophils mediate the initial angiogenic switch in a mouse model of multistage carcinogenesis. Proc Natl Acad Sci USA 103, 12493-12498, 2006.

Okamura Y, Ashida R, Ito T, Sugiura T, Mori K, Uesaka K. Preoperative neutrophil to lymphocyte ratio and prognostic nutritional index predict overall survival after hepatectomy for hepaticellular carcinoma. World J Surg 39, 1501-1509, 2015.

Seretis C, Gourgiotis S, Gemenetzis G, Seretis F, Lagoudianakis E, Dimitrakopoulos G. The significance of neutrophil/lymphocyte ratio as a possible marker of underlying papillary microcarcinoma in thyroidal goiters: a pilot study. Am J Surg 205, 691-696, 2013.

Song Y, Liu H, Gao L, Liu X, Ma L, Lu M, Gao Z. Preoperative neutrophil-to-lymphocyte ratio as prognostic predictor for hypopharyngeal squamous cell carcinoma after radical resections. J Craniofac Surg 26, e137-e140, 2015.

Szkandera J, Gerger A, Liegl-Atzwanger B, Stotz M, Samonigg H, Friesenbichler J, Stojakovic T, Leithner A, Pichler $\mathrm{M}$. The derived neutrophil/lymphocyte ratio predicts poor clinical outcome in soft tissue sarcoma patients. Am J Surg 210, 111-116, 2015.

Turkmen K, Erdur FM, Ozcicek F, Ozcicek A, Akbas EM, Ozbicer A, Demirtas L, Turk S, Tonbul HZ. Platelet-to-lymphocyte ration better predicts inflammation than neutrophil-to-lymphocyte ratio in end-stage renal disease patients. Hemodial Int 17, 391-396, 2013. 
Tuttle RM. Differentiated thyroid cancer: Overview of management. UpToDate, Wolters Kluwer (Eds. Ross DS, Mulder JE), 2017.

Walsh SR, Cook EJ, Goulder F, Justin TA, Keeling NJ. Neuthrophil-lymphocyte ratio as a prognostic factor in colorectal cancer. J Surg Oncol 91, 181-184, 2005.

Wang S, Zhang Z, Fang F, Gao X, Sun W, Liu H. The neutrophil/lymphocyte ratio is an independent prognostic indicator in patients with bone metastasis. Oncol Lett 2, 735-740, 2011.

Whiteside TL. The tumor microenvironment and its role in promoting tumor growth. Oncogene 27, 5904-5912, 2008. 